\title{
Distribución de Aedes aegypti y Aedes albopictus en Guatemala 2016
}

\author{
Distribution of Aedes aegypti and Aedes albopictus in Guatemala 2016
}

\author{
Manuel Lepe ${ }^{1,3 *}$, Marcela Dávila1, Miriam Canet ${ }^{1}$, Yaimie López², \\ Edson Flores ${ }^{5}$, Amílcar Dávila1, Luis E. Escobar ${ }^{4,5}$
}

\begin{abstract}
${ }^{1}$ Departamento de Investigación y Extensión, Facultad de Medicina Veterinaria y Zootecnia, Universidad de San Carlos de Guatemala, Guatemala. ${ }^{2}$ Centro de Estudios en Salud, Universidad del Valle de Guatemala, Guatemala.

${ }^{3} \mathrm{PhD}$ Program in Conservation Medicine, Facultad de Ecología y Recursos Naturales, Universidad Andres Bello, Santiago, Chile. ${ }^{4}$ Department of Fisheries, Wildlife and Conservation Biology, University of Minnesota, Estados Unidos.

${ }^{5}$ Escuela de Estudios de Postgrado, Facultad de Medicina Veterinaria y Zootecnia, Universidad de San Carlos, Guatemala.
\end{abstract}

*Autor al que se dirige la correspondencia: malepelopez@gmail.com

Recibido: 14 de diciembre 2016 / Revisión: 24 de febrero 2016 / Aceptado: 10 de mayo 2016

\section{Resumen}

T os recientes brotes de zika y chikungunya en Guatemala, y el continuo padecimiento de dengue, hacen neceLsario generar preguntas respecto a la presencia de los vectores de arbovirus, Aedes aegypti y A. albopictus, en este país. Los reportes publicados previamente, señalan la presencia de estos vectores en cuatro departamentos de Guatemala. El objetivo de este estudio fue actualizar los reportes de A. aegypti y A. albopictus en Guatemala para informar con datos robustos a las agencias de salud. Se utilizaron datos del Programa de Enfermedades Transmitidas por Vectores del Ministerio de Salud Pública y Asistencia Social, que contiene principalmente información sobre la presencia de estadios larvales de los vectores. Los sitios de colecta de los mosquitos fueron validados usando herramientas de Sistemas de Información Geográfica, generando mapas de distribución de ambas especies de vectores en todo el país. Los resultados incluyen la presencia de A. aegypti en 21 departamentos y A. albopictus en 11. Aedes aegypti fue reportado en zonas bajas con tolerancias a altas temperaturas $\left(32.3-34.8^{\circ} \mathrm{C}\right)$, mientras A. albopictus se reportó en zonas con mayor precipitación y en mayores altitudes $(2,301 \mathrm{~m})$. Se concluye en un notable incremento en la distribución de estas dos especies en Guatemala, basados en los informes del primer trimestre 2016 del Programa de Enfermedades Transmitidas por Vectores colectados en época seca. Se discuten la oportunidad de integrar a la academia con los programas actuales de vigilancia epidemiológica para abordar las necesidades de investigación de estos vectores a nivel nacional.

Palabras claves: Arbovirus, Mosquitos vectores, Vectores de enfermedades, Valores de referencia, Sistemas de información geográfica, Vigilancia epidemiológica

\section{Abstract}

$\mathrm{R}$ ecent outbreaks of zika and chikungunya in Guatemala, and the continued burden of dengue, make necessary to generate novel questions regarding the presence of arboviruses vectors, Aedes aegypti and A. albopictus, in this country. Reports published before this manuscript, indicated the presence of these vectors in four departments of Guatemala. The aim of this study was to update the reports of A. aegypti and A. albopictus in Guatemala to better inform health agencies. We used data from the Programa de Enfermedades Transmitidas por Vectores, which mainly contains information about the presence of larval stages of vectors. Sites of mosquito collection were validated using geographic information systems tools, generating maps of both vector species' occurrence across the country. The results include A. aegypti presence in 21 departments and A. albopictus in 11. Aedes aegypti was reported in lowlands with high temperature tolerance, while A. albopictus was reported in drier zones and in higher altitudes $(2,301 \mathrm{~m})$. We conclude a notable increase in the distribution of these two species in Guatemala, based on the reports of the first quarter of 2016 of the Program of Vector-borne Diseases collected during the dry season. We discuss the opportunity to integrate the academy with epidemiological surveillance programs to address the research needs of these vectors at the national level.

Keywords: Arbovirus, Mosquitoes vectors, Disease vectors, Reference values, geographical information systems, epidemiological surveillance 


\section{Introducción}

Los vectores Aedes aegypti y A. albopictus son objeto de análisis espaciales en todo el mundo, debido a su vinculación en la transmisión de los virus del dengue, chikungunya, zika y fiebre amarilla, y a la necesidad de reconocer los sitios en donde estas especies ocurren para el diseño de planes de control y prevención (Kraemer et al., 2015; Nsoesie, 2016). A nivel mundial, las infecciones por arbovirus representan una alta carga de morbilidad, discapacidad asociada y mortalidad (LaBeaud, Bashir, \& King, 2011). Dengue es la infección arboviral más frecuente con un aproximado de 100 millones de casos anuales, 120 países afectados y un $50 \%$ de la población en riesgo (Olivera-Botello et al., 2016). En Guatemala, de acuerdo a los datos publicados por el Centro Nacional de Epidemiología (CNE), a la semana 52 del 2015 la tasa de infección de dengue fue 111.6 por 100,000 habitantes, 189.9 para chikungunya y 1.24 para zika (CNE, 2016). Lo anterior estimado para la población nacional podría representar al menos 16 mil, 28 mil y 186 personas infectadas con dengue, chikungunya y zika, respectivamente (Instituto Nacional de Estadística [INE], 2012). Esto representa dramáticas pérdidas económicas en términos de días perdidos por enfermedad y costos para Guatemala de entre US $\$ 32.60$ a US\$165.72 por paciente (Zubieta-Zavala et al., 2016).

Sin embargo, información sobre la distribución espacial de los vectores de arbovirus es irregular y escasa a nivel mundial (Bhatt et al., 2013). De hecho, los reportes de A. aegypti y A. albopictus en Guatemala se remontan a los años 1983 (Clark-Gil \& Darsie), 1999 (Monroy et al.) 1996 (Ogata \& López). Otros estudios que reportan indirectamente la presencia en Guatemala, fueron realizados por Usuku y colaboradores (2001), determinando que las prevalencias durante los años 1996 a 1997 están genéticamente relacionadas al virus 3 de dengue, y Rizzo y colaboradores (2012), redujeron poblaciones de Aedes spp. con insecticidas en sitios de cría. Museos de historia natural e institutos de investigación de todo el mundo (e.g., The Global Biodiversity Information Facility [GBIF]) intentan incrementar los datos de ocurrencias para fortalecer la validez y confiabilidad de análisis espaciales (Smith, Johnston, \& Lücking, 2016). Los análisis geográficos que buscan explicar la distribución geográfica de las especies de vectores, su ecología y epidemiologia, utilizan los reportes de presencia y ausencia de las especies en los países afectados (Fatima, Atif, Rasheed, Zaidi, \& Hussain, 2016). Los reportes de ocurrencias permiten un mejor entendimiento de las regiones donde se deben priorizar los recursos para la investigación y prevención de las enfermedades (Koch et al., 2016). Los supuestos de ausencia de los vectores en una región pueden sesgar los análisis de estas enfermedades a nivel local.

En Guatemala, el Ministerio de Salud Pública y Asistencia Social (MSPAS) junto al Programa de Enfermedades Transmitidas por Vectores (ETV) realizan vigilancia entomológica a través de la encuesta sistemática a nivel nacional (MSPAS, 1997). El cuestionario del MSPAS calcula índices entomológicos de densidad y productividad del vector (larvas de mosquito en cuarto estadio). Los indicadores utilizados son índice de vivienda, índice de recipiente, índice de recipiente no útil, índice de pupas e índice de Breteau. Dicha actividad se realiza a través de una encuesta que abarca época seca (noviembre a abril) y época lluviosa (mayo a octubre) verificando la información una semana después (MSPAS, 2015). Para una localidad que posee 1,000 casas, se visita un mínimo de 57, revisando un hogar con intervalos de 18 casas e identificándolas para la verificación semanal y en próximos años. La metodología detallada de la encuesta larvaria se encuentra disponible en el Manual operativo de vigilancia y control entomológico de Aedes aegypti (MSPAS, 2015). De forma breve, las muestras se obtienen utilizando un larvero de malla fina, colocando el material en un recipiente de color blanco para captar por medio de una pipeta las larvas y transportarlas en tubos con $3 \mathrm{ml}$ de alcohol al 70\% para su posterior identificación en el Laboratorio de Entomología. Los registros de dicha vigilancia pueden utilizarse para obtener información espacial sobre los sitios de presencia de los vectores en todo el país (Palaniyandi, 2014a).

Sin embargo, los datos generados por el MSPAS y ETV carecen en su mayoría de coordenadas geográficas, registrando en su mayoría los nombres locales de los sitios (departamento, municipio, aldea, caserío, colonia, entre otros). Parte de esta información puede ser trasladada al sistema de posicionamiento global, construyendo reportes de presencias válidas (Palaniyandi, 2014b). El propósito del presente trabajo fue compilar datos de presencia de las especies de vectores $A$. aegypti y A. albopictus en Guatemala, elaborar una base de datos de presencia de estas especies que pueda ser incluida en análisis espaciales nacionales y mundiales, y finalmente estudiar las condiciones climáticas donde están presentes las especies, utilizando la base de datos publicada por Hijmans, Cameron, Parra, Jones y Jarvis (2005). 


\section{Materiales y métodos}

Se solicitaron al MSPAS los datos de vigilancia entomológica nacional de larvas de A. aegypti y A. albopictus, obtenidas durante el primer trimestre del 2016 (enero, febrero y marzo). Se examinaron los 22 informes recibidos, correspondientes a los 22 departamentos del país, extrayendo los datos que señalaran los sitios de presencia de larvas de los vectores (Araújo \& Williams, 2000). Se ingresó en una matriz los siguientes datos: género y especie colectada, nombre del departamento, municipio, aldea, caserío y localidad de colecta. Los reportes que no cumplieron con la información necesaria anteriormente descrita o que eran duplicados fueron excluidos. Debido a que se presentaron una gran cantidad de datos duplicados se descartó el 91\% de los datos. La información geográfica de los reportes fue validada por medio de la herramienta gratuita Address Validation Tool (2016) que utiliza la descripción del sitio (número y nombre de calle o avenida, ciudad, municipio, departamento, país) y asigna las coordenadas en el mapa que son verificables por el usuario, generando finalmente un archivo de datos delimitados por comas (.csv) incluyendo las coordenadas geográficas en grados decimales (Brotons, Thuiller, Araújo, \& Hirtzel, 2004; Pearce \& Boyce, 2006). Cuatro departamentos presentaron bases de datos con coordenadas geográficas (El Petén, San Marcos, Jalapa y El Progreso). La información de los demás departamentos fue trasladada. Las coordenadas fueron proyectadas en un software de sistemas de información geográfica de acceso libre de nombre Quantum GIS, en un mapa por especie con los límites políticos y divisiones departamentales de Guatemala, obtenido de la base de archivos de la Secretaría de Planificación y Programación de la Presidencia (Segeplan) en formato de archivo vectorial.

Finalmente se establecieron los rangos climáticos tolerados por las especies según las condiciones mensuales de temperatura y precipitación en los sitios de los reportes. Esto es posible, debido a que otros autores han publicado valores para variables climáticas para todo el planeta (por cada mes del año), por consiguiente, las coordenadas geográficas de presencia de una especie se pueden usar para colectar los datos históricos del clima en cada sitio de reporte. Específicamente, se utilizaron las coordenadas de los reportes para extraer los valores climáticos de variables bioclimáticas según Hijmans y colaboradores (2005), disponibles en el repositorio de WorldClim con una resolución espacial aproximada de $18 \mathrm{~km}$, incluyendo valores de elevación en metros sobre el nivel del mar a partir de un cuadro de 250 $\mathrm{m}$ de resolución espacial (Jarvis, Reuter, Nelson, \& Guevara, 2008). Se calculó media, moda, mediana, desviación estándar, valores máximos y mínimos de los datos de las siguientes variables: precipitación anual, temperatura media anual, temperatura máxima anual y temperatura mínima anual.

\section{Resultados}

Los reportes presentaron un rango de datos desde cero (Totonicapán), hasta 493 datos (El Petén) de sitios con presencia de Aedes por departamento. Las bases de datos por procedimiento del ETV-MSPAS incluían los siguientes índices entomológicos: índice de casas, índice de recipientes útiles, índice de recipientes no útiles, índice de pupas e índice de Breteau. Además, se describe el lugar de la pesquisa, pero esta información careció de estandarización en las bases de datos. Por ejemplo, solo algunas bases fueron detalladas al presentar el nombre de la persona que realizó la encuesta, la fecha en día-mes-año, coordenadas geográficas, tipo de criadero (e.g. tonel, llanta, pila), especie identificada y dirección domiciliar exacta del sitio. Se sugiere que el ETV-MSPAS diseñe una boleta única de toma de datos, similar a la boleta incluida en el Manual operativo de vigilancia y control entomológico de Aedes aegypti (MSPAS, 2015), pero agregando coordenadas geográficas y un orden de los datos a escala espacial, donde se incluya primero la dirección domiciliar, y segundo los nombres de pequeña a gran escala: colonia, caserío, aldea, comunidad, municipio, y departamento. Se obtuvieron 202 ocurrencias de larvas para A. aegypti y 75 para $A$. albopictus. De estas ocurrencias fue posible trasladar al sistema GPS 133 reportes de A. aegypti (Figura 1) y 75 de A. albopictus (Figura 2). Once departamentos carecieron de reportes de A. albopictus. Totonicapán fue el único departamento que ETV-MSPAS reportó con ausencia de ambas especies.

La evaluación de las condiciones climáticas de los sitios de ocurrencia, reveló que A. aegypti puede encontrarse en sitios con precipitación anual acumulada de $>2,000 \mathrm{~mm}$ y $A$. albopictus $>2,425 \mathrm{~mm}$. Según valores mensuales, reportes de $A$. aegypti ocurrieron en sitios con temperaturas mínimas de hasta $1^{\circ} \mathrm{C}$ y altas máximas de hasta $34.8^{\circ} \mathrm{C}$ (Tabla 1). Para A. albopictus, tolerancias climáticas incluyeron reportes en sitios con temperaturas mensuales de entre 8.1 y $29.8^{\circ} \mathrm{C}$. Ambas especies de vectores coincidieron en una precipitación mensual bimodal con picos en junio y septiembre, sin 

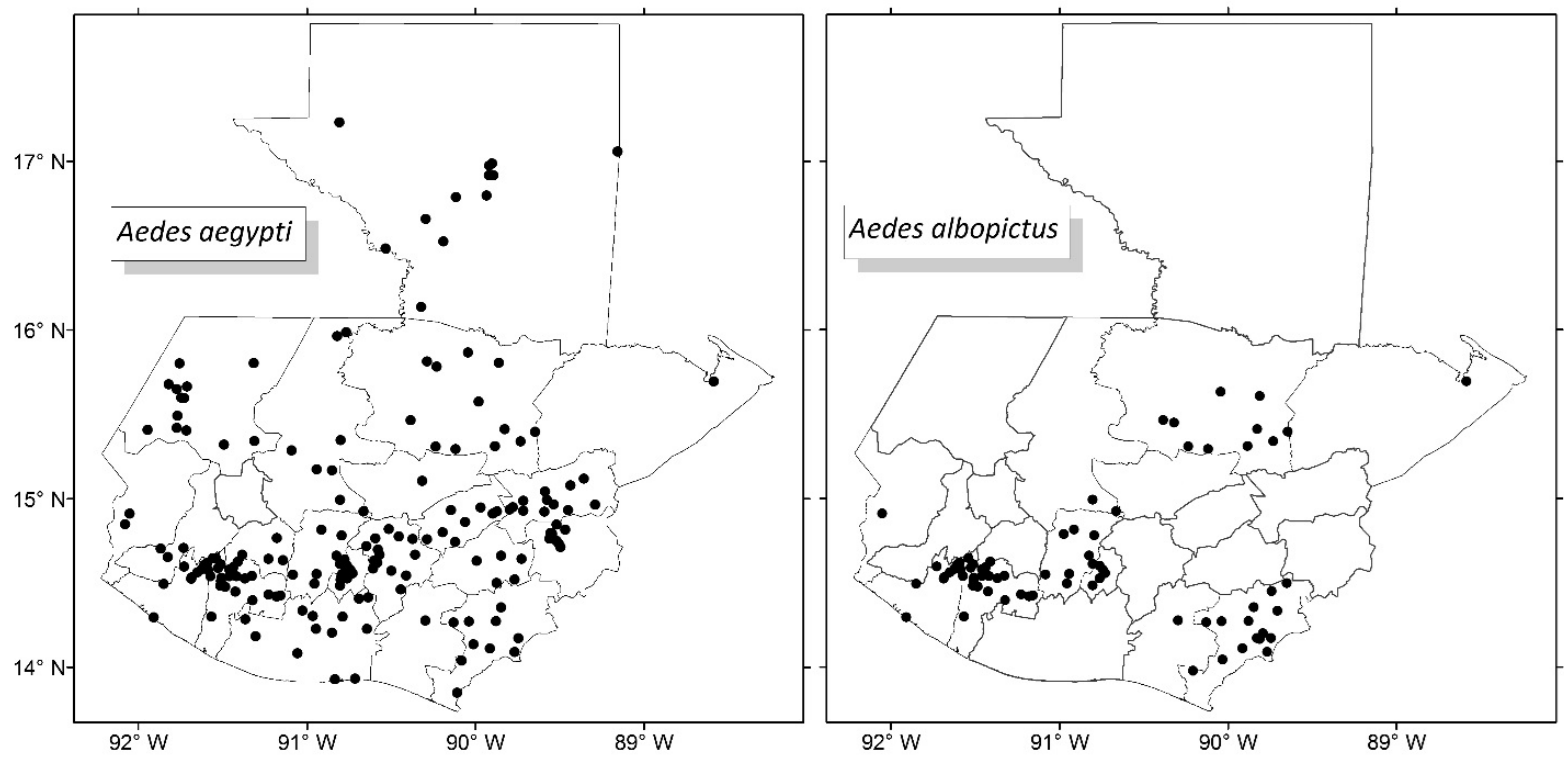

Figura 1. Presencia de vectores de arbovirus en Guatemala. Izquierda: Localización geográfica de Aedes aegypti (puntos negros) en 21 de los 22 departamentos del país. Totonicapán fue el único departamento en donde no se reporta esta especie de vector a la fecha. Derecha: Reportes de Aedes albopictus (puntos negros). No se encontraron reportes para esta especie no fueron encontrados en 11 departamentos de Guatemala.
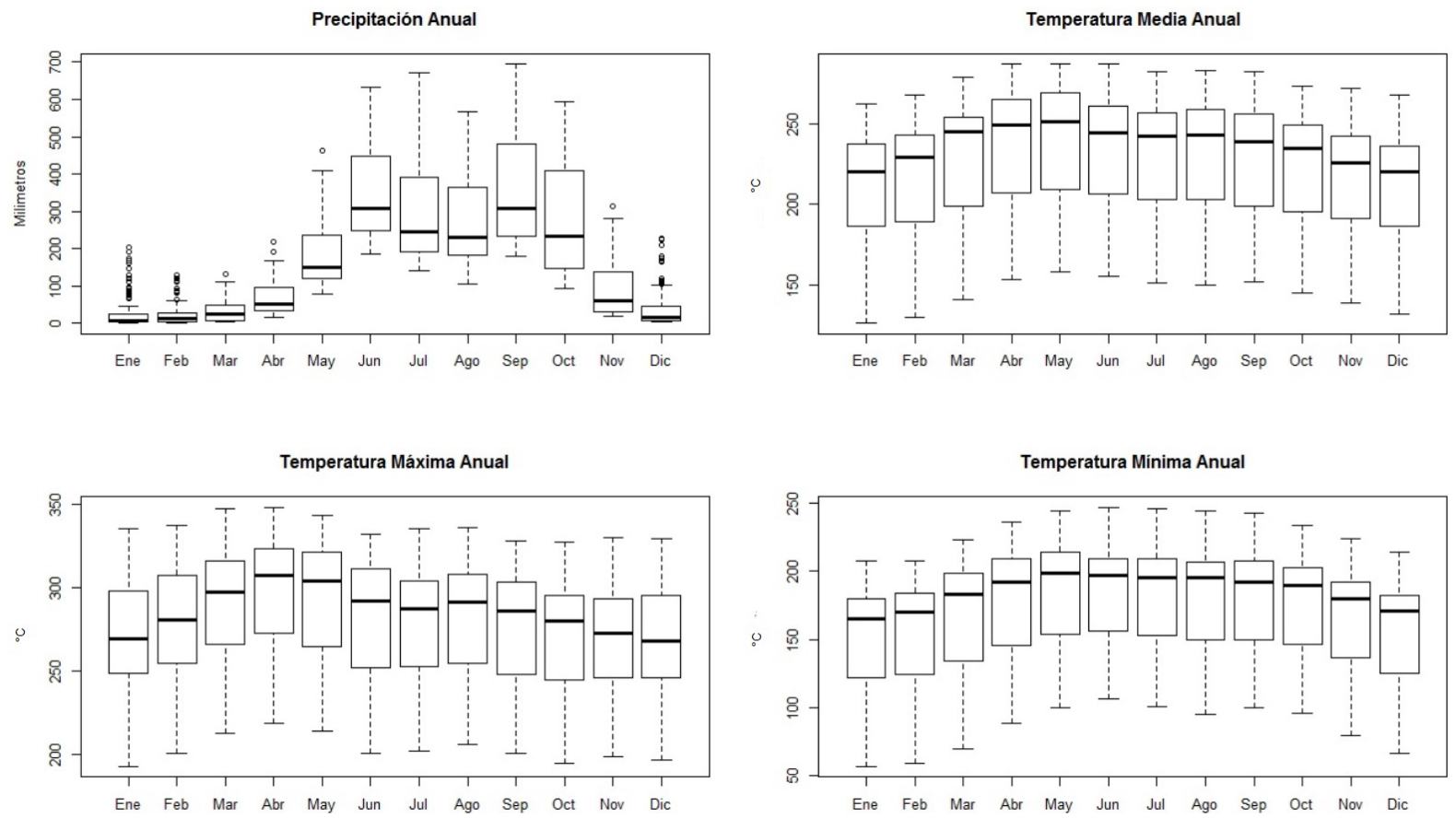

Figura 2. Reporte de precipitación y temperatura durante los meses de reporte de A. aegypti a condiciones de clima en Guatemala. Valores mensuales según todas las presencias reportadas para la especie, mostrando las medianas(barras negras) y error estándar de la media (líneas). 
Tabla 1

Rango de tolerancia a condiciones de temperatura y precipitación y de elevación para A. aegypti y A. albopictus según localización de los reportes de cada especie

\begin{tabular}{ccccc}
\hline Vector & $\begin{array}{c}\text { Precipitación } \\
\text { mensual }(\mathrm{mm})\end{array}$ & $\begin{array}{c}\text { Temperatura Máxima } \\
\left({ }^{\circ} \mathrm{C}\right)\end{array}$ & $\begin{array}{c}\text { Temperatura Mínima } \\
\left({ }^{\circ} \mathrm{C}\right)\end{array}$ & $\begin{array}{c}\text { Elevación } \\
(\mathrm{msnm})\end{array}$ \\
\hline A. aegypti & $1-695$ & $32.3-34.8$ & $5.7-10.7$ & $7-2054$ \\
A. albopictus & $0-695$ & $27.3-29.8$ & $8.1-24.7$ & $10-2301$ \\
\hline
\end{tabular}

embargo, $A$. aegypti fue reportado en sitios con la más baja precipitación (i.e., $23.1 \mathrm{~mm}$; Figura 2) mientras A. albopictus fue reportado en sitios con mayores precipitaciones mensuales (i.e., $426.8 \mathrm{~mm}$; Figura 3). En términos de temperatura, A. aegypti mostró un rango de tolerancia mayor, pero en ambas especies se observó distribución en sitios en donde las más bajas temperaturas ocurren de diciembre a febrero y los meses más cálidos son abril a junio (Figuras 2 y 3). Los reportes de $A$. aegypti representaron las más bajas altitudes (i.e., $7 \mathrm{msnm}$ ) mientras A. albopictus ocurre en las mayores altitudes (i.e., $>2,300 \mathrm{msnm}$; Tabla 1).

\section{Discusión}

Se reporta la presencia de $A$. aegypti en 21 departamentos de Guatemala y A. albopictus en 11 departamentos (Figura 1). Los datos de los reportes del MSPAS que no contenían la información necesaria para ser validados a coordenadas geográficas, fueron excluidos, por lo que la presencia puede ser más amplia de lo reportado aquí. Adicionalmente, algunos departamentos del país señalaron en sus reportes que los datos corresponden a época seca, por lo que pueden existir ocurrencias de mosquitos durante época lluviosa no capturadas en este estudio. Es de recordar que Clark-Gil y Darsie (1983), reportaron para el 1983 la presencia de A. aegypti en los departamentos de Escuintla, Izabal y Retalhuleu. Ogata y Lopez (1996) reportaron colonias de A. albopictus en el departamento de Izabal, y posteriormente Monroy y colaboradores (1999), reportaron poblaciones de esta especie en el mismo departamento.

La información generada en este estudio además de ampliar la geografía de presencia para ambas especies, sugiere que se puede aprovechar el esfuerzo del ETV-MSPAS para incluir registros geográficos que pretendan responder futuros sitios de presencia de los vectores. Debido a que el personal del programa visita todos los municipios y departamentos del país, se deberían equipar con sensores remotos para tomar coordenadas decimales de latitud y longitud de cada sitio de muestreo. Lo anterior permite mayor rigurosidad en próximos esfuerzos de investigación sobre reportes de presencia de vectores. Además, un aumento en el número y distribución espacial y temporal de los sitios de monitoreo puede mejorar la generación de datos que informen a los sistemas de alerta temprana. Finalmente, sería de gran beneficio para la salud pública de Guatemala, una coordinación entre universidades y agencias de salud, para aumentar la difusión de la información utilizando todos los medios posibles tales como congresos, seminarios, revistas arbitradas, cursos universitarios, y capítulos de libros.

Los reportes y distribución de ocurrencia de $A$. aegypti y A. albopictus se incrementaron, contribuyendo a la identificación de sitios en donde se suponía su ausencia. Es posible que esta información no sea desconocida para el MSPAS, pero es necesario que investigadores asociados a universidad se involucren para publicarla en conjunto a la comunidad científica. Ruiz-López y colaboradores (2016) reportaron la presencia de $A$. aegypti en sitios con alturas de 2,302 msnm en Colombia, los reportes de este estudio fueron por debajo de esos valores (i.e., 2,054 $\mathrm{msnm}$ ), lo que sugiere que deben incrementarse muestreos en zonas altas. Carvajal y colaboradores (2016), señalaron la presencia de $A$. albopictus para nuevas áreas de Colombia mientras Requena-Zuñiga, Mendoza y Guevara (2016) y Troyes, Villegas y Troyes (2016) recientemente registraron nuevas áreas de distribución de $A$. aegypti en Perú. Esto sugiere que las zonas que se reportan aquí con ausencia de reportes deben considerarse con precaución: mientras los sitios con presencia de $A$. aegypti son confiables considerando que la identificación es respaldada por análisis de laboratorio, las ausencias de reportes no necesariamente reflejan que la especie 

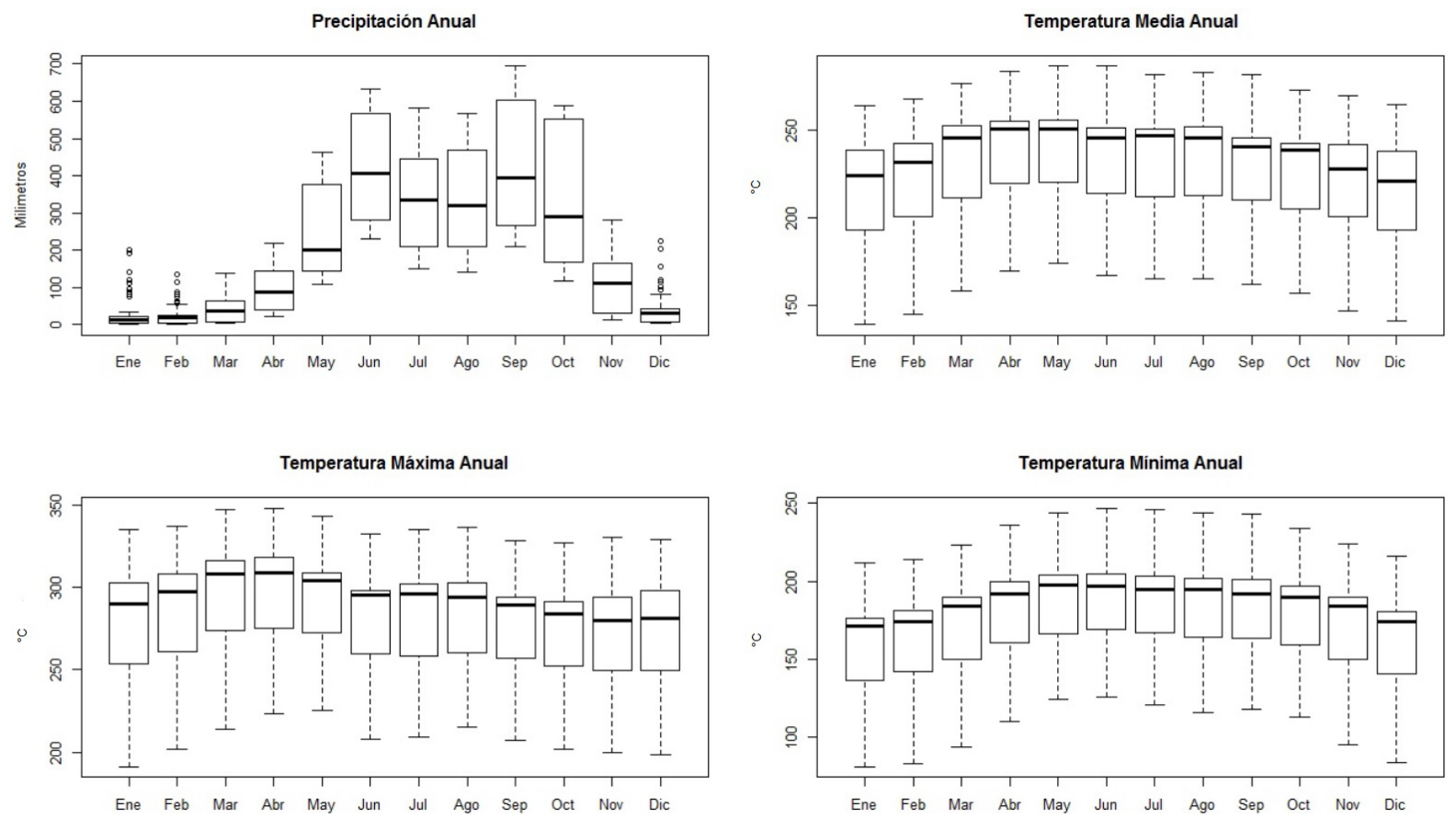

Figura 3. Reporte de precipitación y temperatura durante los meses de reporte de Aedes albopictus a condiciones de clima en Guatemala. Valores mensuales según todas las presencias reportadas para la especie, mostrando las medianas(barras negras) y error estándar de la media (líneas).

no esté presente y puede simplemente corresponder a vacíos de información.

Los recientes reportes de A. albopictus en varios sitios de América Latina sugieren una reciente dispersión de esta especie a sitios antes desocupados o mejoramiento en los sistemas de colecta e identificación de mosquitos. Mentor, Diéguez, Pérez y Vázquez (2003) reportaron por primera vez A. albopictus en Camagüey, Cuba, y recientemente Marín-Rodríguez y colaboradores (2014) reportaron los primeros registros de A. albopictus en áreas metropolitanas de Costa Rica. En el caso específico de los datos del ETV-MSPAS, se puede decir que es una fuente confiable, pues otros autores reportan para dicho programa capacidad tradicional en materia de control de vectores (Nakagawa, Kojima, Zúñiga, Ota, \& Serpas, 2006) y estructura institucional para la vigilancia del género Aedes iniciada en la década de 1960 (San Martín \& Brathwaite-Dick, 2007). Por otra parte, la técnica del cucharón (Organización Panamericana de la Salud [OPS], 1995), utilizada para la toma de larvas y su posterior trasladado al Laboratorio de Entomología del Programa ETV-MSPAS, es una técnica sencilla y adecuada para identificación de especies por características morfológicas. Considerando la confiabilidad de estos métodos, que nuestros datos fueron colectados de fuentes oficiales y que encontramos que la literatura científica sobre Aedes en Guatemala esta desactualizada, este estudio sugiere que los esfuerzos de vigilancia de las agencias de salud de Guatemala podrían ser potencializados en conjunto con los centros de investigación de las universidades. Esto es particularmente importante considerando que las decisiones en salud pública pueden debilitarse por separación con la academia y viceversa. Por ejemplo, el Centro para la Prevención de Enfermedades de Estados Unidos (CDC) recientemente ha recomendado viajar a zonas de Latino América con altitudes superiores a los 2,000 m sugiriendo que en esas zonas no existe riesgo de Zika virus pues los vectores (i.e., Aedes) no se reportan en estas altitudes (Cetron, 2016). Nosotros encontramos reportes de los vectores de Zika en altitudes muy superiores a los 2,000 m (Tabla 1), mostrando la importancia de la actualización de los reportes de distribución de los vectores en la inteligencia en salud y apoyando previos modelos teóricos contradiciendo las recomendaciones del CDC (Peterson, Qiao, \& Escobar, 2016). 
En el caso de Guatemala, previo a este trabajo, los reportes de A. albopictus son escasos y antiguos, incluyendo únicamente el departamento de Izabal, y reportando ausencia en ciudad de Guatemala, Tecún Umán en el departamento San Marcos, y Puerto Quetzal en el departamento Escuintla (Ogata \& López, 1996; Tabaru et al, 1998). Los datos del ETV-MSPAS sugieren una ampliación de la presencia de $A$. albopictus en el país, coincidiendo con los reportes de presencia esta especie en Chiapas, México (Martínez \& Estrada, 2003) geográficamente vecino de San Marcos, Guatemala. Se muestra una actualización de la presencia de ambas especies de vectores para Guatemala con resultados que respaldan una ampliación en el rango de distribución de ambos vectores en términos de área y elevación. Estos datos deben ser tomados con cautela, pero pueden ayudar a diseñar futuros estudios ecológicos y epidemiológicos en las nuevas zonas de registro.

Kraemer y colaboradores (2015) liberaron una impresionante base de datos de ocurrencias de $A$. aegypti y A. albopictus a nivel mundial. En dicha base de datos, Guatemala posee alrededor de 14 ocurrencias de ambas especies en solo cuatro departamentos (Escuintla, Izabal, Retalhuleu y Guatemala). La base de datos del presente estudio, señala 208 ocurrencias únicas (i.e., sin duplicados) para ambas especies, con ausencia de reportes en un solo departamento del país (i.e., Totonicapán). Bajo este escenario, los mapas de distribución propuestos por Kraemer y colaboradores (2015) para Guatemala son incompletos.

Los resultados presentados pueden contribuir en el desarrollo de análisis espaciales para dengue, chikungunya, y zika en Guatemala (Peterson, Sánchez-Cordero, Beard, \& Ramsey, 2002). Conocer el sitio exacto en donde una especie puede encontrar condiciones habitables permite predecir la distribución de las especies. Por ejemplo, determinar los rangos temporales de temperaturas y precipitación permitirían predecir zonas en Guatemala que cumplen con las condiciones climáticas ideales para la ocurrencia de mosquitos (Peterson, 2001). Aquí, presentamos una caracterización de las tolerancias de temperatura, precipitación y elevación para cada uno de los vectores, y mostramos que mientras $A$. aegypti tiene una mayor tolerancia a zonas cálidas, $A$. albopictus se puede encontrar a mayores altitudes y en zonas más húmedas.

La vigilancia entomológica del programa ETVMSPAS puede continuar informando sobre la distribución de estos y otros vectores (Rodríguez, 2002). Sin embargo, es necesario un manejo adaptativo conside- rando estos resultados. Aparentemente, todos los años se muestrean los mismos sitios de vigilancia a nivel de la república (MSPAS, 2015). Con la información generada en este estudio, es posible guiar futuros planes de monitoreo en sitios reportados con supuesta ausencia de las especies, por ejemplo en el departamento de Totonicapán podrían muestrearse las áreas colindantes con sitios de presencia, principalmente con el departamento de Sololá. La determinación de los sitios con ausencia estricta, son fundamentales para describir las condiciones bióticas y abióticas que pueden explicar la falta de éxito biológico de los vectores, lo que puede ayudar a diseñar métodos de control (Miller \& Holloway, 2015).

Aunque la vigilancia epidemiológica en Guatemala se realiza con base en los límites políticos de los departamentos, es necesario abordar el problema basándose en bordes ecológicos a nivel nacional (Hahn et al., 2016). Por ejemplo, Totonicapán puede reportar presencia de vectores en el corto plazo considerando que los departamentos vecinos reportan la presencia de A. aegypti y $A$. albopictus. Se propone que la vigilancia activa en Totonicapán, puede realizarse en los límites territoriales de otros departamentos con abundantes reportes de los vectores (e.g., Huehuetenango).

La información epidemiológica acerca de los casos clínicos de dengue, zika y chikugunya, más allá de los sitios con reporte de vectores (Figura 1), respalda la idea de vacíos epidemiológicos con respecto al monitoreo de la presencia de A. aegypti y A. albopictus (CNE, 2016). Según el CNE, los departamentos con mayor cantidad de casos de enfermedades arbovirales son: Zacapa, Guatemala, Quetzaltenango, Retalhuleu, Jutiapa, Izabal, Santa Rosa, Suchitepéquez y Escuintla. Los mapas de ocurrencia de A. aegypti y A. albopictus (Figura 1) coinciden con lo reportado por el CNE. Sin embargo, en Totonicapán, que se propone como un sitio con ausencia de los vectores, existen reportes de casos clínicos de arbovirosis (i.e., CNE reportó 6 casos de dengue en 2014 y 5 casos en 2015). La información de los casos de enfermedad (anamnesis y pruebas de laboratorio), no permite esclarecer el sitio de infección y su etiología. Es decir, los datos colectados como parte de la historia clínica del paciente, no esclarecen si se infecta en otra región del país (registrando los lugares visitados en el último mes), y si estos casos reportados como dengue, pueden ser causados por otros arbovirus.

Las condiciones ambientales en donde se reporta la presencia de ambas especies de Aedes deben ser sujeta a análisis poblacional (Steffler et al., 2016). En el presente caso se reportan los valores climáticos de 
las variables propuestas por Hijmans y colaboradores (2005), de cada una de los reportes validados. Esto no es sugerente de que dichos rangos sean los apropiados para el desarrollo, crecimiento y supervivencias de las especies, en su lugar, son patrones de clima que explican la macroecología de zonas con exitosa reproducción de las especies. Jansen y Bebee (2010) señalan que además de los patrones de clima a gran escala, es necesario indagar los microambientes domésticos para complementar las aproximaciones de distribución. Podría generarse estudios experimentales para verificar si los valores reportados aquí tienen efecto en las poblaciones de mosquitos en Guatemala (Hopp \& Foley, 2001).

Las ocurrencias de A. aegypti y A. albopictus son un insumo para aumentar la comprensión del impacto de los arbovirus presentes en Guatemala (Beck, Böller, Erhardt, \& Schwanghart, 2014). Es necesario indagar otros elementos que pudieran verse implicados a escalas de microclima (e.g. temperatura, humedad relativa, oxígeno disuelto, y turbidez del agua). Además de esfuerzos por entender los aspectos culturales en la población humana de Guatemala en respuesta a estas enfermedades (Bastos \& Camus, 2004), que puede influir en la presencia y ausencia de los vectores. Estos resultados pueden contribuir a entender las tolerancias climáticas, distribución espacial y altitudinal de $A$. aegypti y $A$. albopictus, aportando a los esfuerzos por disminuir la transmisión de enfermedades arbovirales en Guatemala, con base a aspecto biogeográficos de estos vectores.

Existen limitantes en las proposiciones que se desarrollan en el presente estudio con respecto a la distribución desde un punto de vista ecológico. Los datos de época lluviosa complementarían la distribución propuesta, debido a que podría modificarse con el aumento de la abundancia de los mosquitos al momento de mayor precipitación. Por otra parte, la distribución planteada es a gran escala, por lo que es necesario continuar con esfuerzos de investigación a una escala menor. Por ejemplo, la información generada por índices entomológicos podría ser abordada para determinar patrones antropogénicos asociados a la abundancia de los vectores. Además es necesario conocer los patrones culturales que propician la abundancia, principalmente en áreas urbanas y rurales. Lo anterior se resume en la necesidad de valorar los datos de la vigilancia epidemiológica del ETV-MSPAS, para su fortalecimiento por parte de la academia, proponiendo análisis que puedan determinar los factores que explican la presencia-ausencia, distribución y abundancia de estos vectores a nivel nacional.
Existe una variedad de interrogantes, como consecuencia del reporte de ocurrencias del presente trabajo. Se necesita esclarecer si el departamento de Totonicapán, principalmente en las regiones que colinda con otros departamentos, hay presencia de los vectores. Esto permitiría el estudio y análisis de los factores bióticos y abióticos que provocan su ausencia. Por otra parte, es posible verificar la ausencia de reportes de $A$. albopictus en los varios departamentos, debido su rango de temperaturas, precipitación y altitudes, en otros departamentos sin reportes también existen las características necesarias para su dispersión. Se sugiere continuar con la búsqueda de sitios específicos de presencia y ausencia de los vectores para Guatemala, lo que permitiría un riguroso análisis espacial y su posible correlación con el cambio climático.

\section{Agradecimientos}

Se agradece al personal del Programa ETVMSPAS por facilitar las bases de datos de vigilancia entomológica de los vectores. Esta investigación fue cofinanciada por Digi-Usac-2016, Proyecto:4.8.63.1.86. Al Minnesota Environment and Natural Resources Trust Fund, el Minnesota Aquatic Invasive Species Research Center y el Clean Water Land and Legacy, por financiar a Luis E. Escobar.

\section{Referencias}

Address Validation Tool. (2016). Online maps of Australia: data, information, tools, solutions. Australia. Recuperado de: http://www.aus-emaps.com/ bulk_geocoder.php

Araújo, M. B., \& Williams, P. H. (2000). Selecting areas for species persistence using occurrence data. Biological Conservation, 96, 331-345. doi: 10.1016/S0006-3207(00)00074-4

Bastos, S., \& Camus, M. (2004). Multiculturalismo y pueblos indígenas: reflexiones a partir del caso de Guatemala. Revista Centroamericana de Ciencias Sociales, 1(1), 87-112.

Beck, J., Böller, M., Erhardt, A., \& Schwanghart, W. (2014). Spatial bias in the GBIF database and its effect on modeling species' geographic distributions. Ecological Informatics, 19, 10-15. doi: 10.1016/j.ecoinf.2013.11.002 
Bhatt, S., Gething, P. W., Brady, O. J., Messina, J. P., Farlow, A. W., Moyes, C. L., ... Myers, M. F. (2013). The global distribution and burden of dengue. Nature, 496(7446), 504-507. doi: 10.1038/ nature 12060

Brotons, L., Thuiller, W., Araújo, M. B., \& Hirtzel, A. H. (2004) Presence-absence versus presence-only modelling methods for predicting bird habitat suitability. Ecography, 27, 437-448. doi: 10.1111/j.0906-7590.2004.03764.x

Clark-Gil, S., \& Darsie, R. F. (1983). The mosquitoes of Guatemala. Their identification, distribution and bionomics, with keys to adult females and larvae in English and Spanish. Mosquito Systematics, 15(3), 151-284.

Carvajal, J. J., Honorio, N. A., Díaz, S. P., Ruiz, E. R., Asprilla, J., Ardila, S., \& Parra, G. J. (2016). Detección de Aedes albopictus (Skuse) (Diptera: Culicidae) en el municipio de Istmina, Chocó, Colombia. Biomédica, 36(3), 438-446. doi: 10.7705/ biomedica.v36i3.2805

Centro Nacional de Epidemiologia. (2016). Situación de semanas epidemiológicas 2015. Guatemala. Ministerio de Salud Pública y Asistencia Social. Recuperado de http://epidemiologia.mspas. gob.gt/index.php/dos/salas-situacionales/situacion-de-semanas-epidemiologicas

Cetron, M. (2016). Revision to CDC's Zika travel notices: minimal likelihood for mosquito-borne Zika virus transmission at elevations above 2,000 meters. Morbidity and mortality weekly report (MMWR), 65(10), 267-268

Fatima, S. H., Atif, S., Rasheed, S. B., Zaidi, F., \& Hussain, E. (2016). Species distribution modelling of Aedes aegypti in two dengue-endemic regions of Pakistan. Tropical Medicine \& International Health, 21(3), 427-436. doi: 10.1111/tmi.12664

Hahn, M. B., Eisen, R. J., Eisen, L., Boegler, K. A., Moore, C. G., McAllister, J., ... Mutebi, J. P. (2016). Reported distribution of Aedes (Stegomyia) aegypti and Aedes (Stegomyia) albopictus in the United States, 1995-2016 (Diptera: Culicidae). Journal of Medical Entomology, 53(5), 1169-1175. doi: 10.1093/jme/tjw072

Hijmans, R. J., Cameron, S. E., Parra, J. L., Jones, P. G., \& Jarvis, A. (2005). Very high resolution interpolated climate surfaces for global land areas.
International Journal of Climatology, 25(15), 1965-1978. doi: 10.1002/joc.1276

Hopp, M. J., \& Foley, J. A. (2001). Global-scale relationships between climate and the dengue fever vector, Aedes aegypti. Climatic Change, 48(2-3), 441-463. doi:10.1023/A:1010717502442

Instituto Nacional de Estadística. (2012). Caracterización estadística, Republica de Guatemala 2012. Recuperado de https://www.ine.gob.gt/sistema/ uploads/2014/02/26/5eTCcFlHErnaNVeUmm3iabXHaKgXtw0C.pdf

Jarvis, A., Reuter, H. I., Nelson, A., \& Guevara. E. (2008). Hole-filled SRTM for the globe Version 4, available from the CGIAR-CSI SRTM 90m Database. Recuperado de http://srtm.csi.cgiar.org

Jansen, C. C., \& Beebe, N. W. (2010). The dengue vector Aedes aegypti: what comes next. Microbes and Infection, 12(4), 272-279.

Koch, L. K., Cunze, S., Werblow, A., Kochmann, J., Dörge, D. D., Mehlhorn, H., \& Klimpel, S. (2016). Modeling the habitat suitability for the arbovirus vector Aedes albopictus (Diptera: Culicidae) in Germany. Parasitology Research, 115(3), 957-964. doi:10.1007/s00436-015-4822-3

Kraemer, M. U., Sinka, M. E., Duda, K. A., Mylne, A. Q., Shearer, F. M., Barker, C. M., ... Hendrickx, G. (2015). The global distribution of the arbovirus vectors Aedes aegypti and A. albopictus. Elife, 2015(4), 1-17. doi: 10.7554/eLife.08347

LaBeaud, A., Bashir, F., \& King, C. H. (2011). Measuring the burden of arboviral diseases: The spectrum of morbidity and mortality from four prevalent infections. Population Health Metrics, 9(1), 1-11. doi: 10.1186/1478-7954-9-1

Marín-Rodríguez, R., Calderón-Arguedas, O., Díaz Ríos, M., Duarte Solano, G., Valle Arguedas, J. J., \& Troyo Rodríguez, A. (2014). First finding of Aedes albopictus Skuse in the the Greater Metropolitan Area of Costa Rica. Revista Costarricense de Salud Pública, 23(1), 1-4.

Martínez, M. C., \& Estrada, J. L. T. (2003). First evidence of Aedes albopictus (Skuse) in southern Chiapas, Mexico. Emerging Infectious Diseases, 9(5), 606-607.

Miller, J. A., \& Holloway, P. (2015). Incorporating movement in species distribution models. Progress in Physical Geography, 39(6), 837-849. 
Ministerio de Salud Pública y Asistencia Social. (1997). Manual de referencia para la aplicación de las normas de atención: Dengue. Guatemala, Guatemala: Autor.

Ministerio de Salud Pública y Asistencia Social. (2015). Manual operativo de vigilancia y control entomológico de Aedes aegypti. Guatemala, Guatemala: Autor. Recuperado de: http://epidemiologia.mspas.gob.gt/files/Publicaciones \%202016/ Manuales/Manual\%20\%20operativo\%20de\%20 Aedes\%20aegypti\%20(VIRTUAL).pdf

Mentor, V., Diéguez, L., Pérez, E., \& Vázquez, A. (2003). Presencia de Aedes (Stegomyia) albopictus Skuse 1894 (Díptera: Culicidae) en Camagüey. Importante vector de la fiebre del dengue; dengue hemorrágico y del virus del Nilo occidental. Revista Archivo Médico de Camagüey, 7(6), 764-771.

Monroy, C., Yuichiro, T., Rodas, A., Mejía, M., Pichilla, R., Mauricio, H., \& Pérez M. (1999). Distribución de Aedes albopictus (Diptera: Culicidad) en Guatemala, seguimiento a una colonización de 1995. Revista Científica, 12(1), 29-32.

Nakagawa, J., Kojima, M., Zúñiga, C., Ota, \& K., \& Serpas. M. (2003). Modalidades de descentralización y control de la enfermedad de Chagas: Análisis comparativo entre Guatemala, Honduras y El Salvador. En Z. E. Yadón, R. E. Gürtler, F. Tobar \& A. C. Medici (Eds.), Descentralización y gestión del control de las enfermedades transmisibles en América Latina (pp. 231-254). Buenos Aires, Argentina: Organización Panamericana de la Salud.

Nsoesie, E. O., Kraemer, M. U., Golding, N., Pigott, D. M., Brady, O. J., Moyes, C. L., ... \& Hay, S. I. (2016). Global distribution and environmental suitability for chikungunya virus, 1952 to 2015. Eurosurveillance, 21(20), 1-12.

Ogata, K., \& Lopez, S. A. (1996). Discovery of Aedes albopictus in Guatemala. Journal of the American Mosquito Control Association, 12 (3 Pt 1), 503506.

Olivera-Botello, G., Coudeville, L., Fanouillere, K., Guy, B., Chambonneau, L., Noriega, F., ... Chotpitayasunondh, T. (2016). Tetravalent dengue vaccine reduces symptomatic and asymptomatic dengue virus infections in healthy children and adolescents aged 2-16 years in Asia and Latin America. Journal of Infectious Diseases, 214(7), 994-1000. doi: 10.1093/infdis/jiw297

Organización Panamericana de la Salud. (1995). Dengue y dengue hemorrágico en las Américas: Guías para su prevención y control. (Publicación científica No. 548). Washington, D.C: Autor.

Palaniyandi, M. (2014a). The environmental aspects of dengue and chikungunya outbreaks in India: GIS for epidemic control. International Journal of Mosquito Research, 1(2), 38-44.

Palaniyandi, M. (2014b). Web mapping GIS: GPS under the GIS umbrella for Aedes species dengue and chikungunya vector mosquito surveillance and control. International Journal of Mosquito Research, 1(3), 18-25.

Pearce, J. L., \& Boyce, M. S. (2006). Modelling distribution and abundance with presence-only data. Journal of Applied Ecology, 43(3), 405-412.

Peterson, A. T. (2001). Predicting species' geographic distributions based on ecological niche modeling. The Condor, 103(3), 599-605.

Peterson, A. T., Sánchez-Cordero, V., Beard, C. B., \& Ramsey, J. M. (2002). Ecologic niche modeling and potential reservoirs for Chagas disease, Mexico. Emerging Infectious Diseases, 8(7), 662-667.

Peterson, A. T., Osorio, J. E., Qiao, H., and Escobar, L. E. (2016). Zika virus, elevation, and transmission risk. PLoS Current Outbreaks, 1, 1-9.

Requena-Zuñiga, E., Mendoza, L., \& Guevara, M. (2016). Nuevas áreas de distribución de Aedes aegypti en Perú. Revista Peruana de Medicina Experimental y Salud Publica, 33(1), 171-172.

Rizzo, N., Gramajo, R., Escobar, M. C., Arana, B., Kroeger, A., Manrique-Saide, P., \& Petzold, M. (2012). Dengue vector management using insecticide treated materials and targeted interventions on productive breeding-sites in Guatemala. BMC Public Health, 12(1), 1-10. doi: 10.1186/14712458-12-931

Rodríguez, R. (2002). Estrategias para el control del dengue y del Aedes aegypti en las Américas. Revista Cubana de Medicina Tropical, 54(3), 189201.

Ruiz-López, F., González-Mazo, A., Vélez-Mira, A., Gómez, G. F., Zuleta, L., Uribe, S., \& Vélez-Bernal, I. D. (2016). Presencia de Aedes (Stegomyia) 
aegypti (Linnaeus, 1762) y su infección natural con el virus dengue en alturas no registradas para Colombia. Biomédica, 36(2), 303-308. doi: 10.7705/biomedica.v36i2.3301

San Martín, J. L., \& Brathwaite-Dick, O. (2007). La estrategia de gestión integrada para la prevención y el control del dengue en la región de las Américas. Revista Panamericana de Salud Pública, 21(1), 55-63.

Smith, B. E., Johnston, M. K., \& Lücking, R. (2016). From GenBank to GBIF: Phylogeny-based predictive niche modeling tests accuracy of taxonomic identifications in large occurrence data repositories. PLoS ONE, 11(3), 1-15. doi: 10.1371/ journal.pone.0151232

Steffler, L. M., Dolabella, S. S., Ribolla, P. E. M., Dreyer, C. S., Araújo, E. D., Oliveira, R. G., \& La Corte, R. (2016). Genetic variability and spatial distribution in small geographic scale of Aedes aegypti (Diptera: Culicidae) under different climatic conditions in Northeastern Brazil. Parasites \& Vectors, 9(1), 530.

Tabaru, Y., Monroy, C., Rodas, A., Mejia, M., Pichilla, R., Mauricio, H., \& Perez, M. (1998). Distribution of Aedes albopictus (Diptera: Culicidae) in Gua- temala, following colonization in 1995. Medical Entomology and Zoology, 49(4), 331-336.

Troyes, L., Villegas, Z., \& Troyes, M. (2016). Expansión del Aedes aegypti a localidades rurales de Cajamarca. Revista Peruana de Medicina Experimental y Salud Publica, 23(3), 163-167.

Usuku, S., Castillo, L., Sugimoto, C., Noguchi, Y., Yogo, Y., \& Kobayashi, N. (2001). Phylogenetic analysis of dengue-3 viruses prevalent in Guatemala during 1996-1998. Archives of Virology, 146(7), 1381-1390.

Zubieta-Zavala, A., Salinas-Escudero, G., Ramírez-Chávez, A., García-Valladares, L., López-Cervantes, M., Yescas, J. G. L., \& Durán-Arenas, L. (2016). Calculation of the average cost per case of dengue fever in Mexico using a micro-costing approach. PLoS Neglected Tropical Diseases, 10(8), 1-14. doi:10.1371/journal. pntd.0004897 